\title{
THE FREE PRODUCT OF SKEW FIELDS
}

\author{
Dedicated to the memory of Hanna Neumann
}

P. M. COHN

(Received 7 June 1972)

Communicated by M. F. Newman

\section{Introduction}

In a recent paper [3] it was shown that the free product $K * L$ of two fields (possibly skew) can be embedded in a field, and moreover, this latter can be chosen to be the 'universal field of fractions' of $K * L$ (cf. [4,5]). This opens up the prospect of doing for skew fields what the Neumanns and others have done for groups; indeed some sample applications were given in [3]. We pursue this topic here a little further: our main results state (i) every ccuntably generated field can be embedded in a 2-generator field, (ii) in a free prcduct of rings over a field $k$, any element algebraic over $k$ is conjugate to an element in one of the factors, (iii) any field can be embedded in a field with $n$th roots for each $n$. These results are all analogous to well known results in group theory (cf. [8]), and although the proofs are not just a translation of the group case, the latter is of scree help. Thus (ii), (iii) follow fairly easily, but they lead to other problems, still ofen, by replacing 'free product of rings' in (ii) by 'field prcduct of fields, and in (iii) replacing ' $n$th roots' by 'roots of any equation'. On the other hand, (i) is less immediate, since 'field products' need to be used in the proof, and their manipulation requires some more technical lemmas.

I am indebted to G. M. Bergman for his helpful comments on several earlier versions of this note.

\section{The field product}

Throughout, all rings have a unit element 1 , preserved by homomorphisms and inherited by subrings, and fields are not necessarily commutative; occasionally we use the prefix 'skew' for emphasis. If $K$ is any ring, by a $K$-ring we understand a ring $R$ with a canonical homomorphism $K \rightarrow R$; since $R$ has a 1, this is equivalent to imposing on $R$ a $K$-bimodule structure satisfying $x(y z)=(x y) z$, for $x, y, z$ in $R$ or $K$. Given such a structure, the homomorphism is obtained by mapping $\alpha \mapsto \alpha .1$, where $\alpha \in K$ and 1 is the unit element of $R$. A $K$-ring is faithful if the map 
$K \rightarrow R$ is injective; we note that if $K$ is a field, any non-zero $K$-ring is faithful.

Let $k$ be a field and $\left(R_{\lambda}\right)$ a family of non-zero (and hence faithful) $k$-rings. In each $R_{\lambda}$ we choose a right $k$-basis including 1 ; this may be denoted by $B_{\lambda}^{\prime}=\{1\} \cup B_{\lambda}$. We now form the words in $\bigcup B_{\lambda}$; such a word is called non-interacting if neighbouring factors come from different sets $B_{2}$. The set of all noninteracting words (including the empty word to represent 1 ) for ms a right $k$-kasis for the free product of the $R_{\lambda}$ over $k$ (see [1,2」). This free prcduct will be written ${ }_{k}^{*} R_{\lambda}$, or if only two factors $R, S$ are present, $R_{k}^{*} S$.

Although we shall have occasion to deal with the free product directly, most of the time we are interested in the fields that can be formed from it. Thus let $\left(K_{\lambda}\right)$ be a family of fields containing $k$ as a subfield and let $R={ }_{k}^{*} K_{\lambda}$ be their free product; from [2] we know that $R$ is a fir. We recall that a ring $R$ is called a fir ( $=$ free ideal ring) if every (right or left) ideal of $R$ is free as $R$-module, of unique rank. If every finitely generated right (or equivalently, left) ideal is free, of unique rank, $R$ is called a semifir. In [3] it was shown that every fir is embeddable in a field; we therefore have a field $L$ containing all the $K_{\lambda}$. However, a minimal field containing all the $K_{\lambda}$ may not be unique and we shall need the more precise description of the universal field of fractions $[4,5]$, which is uniquely determined. We briefly summarize the construction from [4], which more generally, provides a universal field of fractions for any semifir.

Let $R$ be any ring; by an $R$-field we understand a field $K$ which is an $R$-ring. Such an $R$-field is called epic $\left(^{*}\right)$ if the canonical map $R \rightarrow K$ is a ring-epimorphism. This is equivalent to the condition that $K$ be generated, as a field, by the inage of $R$. A specialization of epic $R$-fields $K, L$ is an $R$-ring homomorphism $f: K_{0} \rightarrow L$ from an $R$-subring $K_{0}$ of $K$ to $L$ such that $x f \neq 0$ implies $x^{-1} \in K_{0}$. It follows from this that $K_{0}$ is a local ring (i.e. its non-units form an ideal, cf. [4]); in fact ker $f$ is the maximal ideal and $K_{0} / \mathrm{ker} f \cong L$. Two specializations are equal if they agree on a common subring $K_{0}$ and the common restriction is a specialization. The epic $R$-fields and specializations can be shown to form a category $\mathscr{F}_{R}$; an initial object in $\mathscr{F}_{R}$, if one exists, is called a universal $R$-field, or in case the canonical map is injective, a universal field of fractions for $R$.

For a commutative ring $R$, the epic $R$-fields are determined up to isomorphism by the kernels of the canonical maps. The collection of these kernels, the prime ideals of $R$, forms a category equivalent to $\mathscr{F}_{R}$ (with inclusions as maps). This is usually written spec $R$ and called the prime spectrum of $R$. In the general case, an epic $R$-field $K$ is not determined by the elements of $R$ that map to zero, but by the set of square matrices over $R$ that become singular over $K$. Thus the place of the prime ideal is taken by a collection of square matrices satisfying certain conditions; this is called a prime matrix ideal, and it is possible to write

(*) This differs slightly from the usage in [4]: what are called $R$-fields there are called epic $R$-fields here. 
down a brief set of conditions characterizing prince matrix ideals [4]. The collection of prime matrix ideals of a general ring $R$ is written field-spec $R$; it is equivalent, as a category, to $\mathscr{F}_{R}$. In particular, it is possible to write down conditions for field-spec $R$ to be non-empty. We shall not give the general conditions here, as they are not needed (see [4], chapter 7 for details), but only note one special case.

A matrix $A$ over a ring $R$ is said to be full if it is square, say $n \times n$, and cannot be written as $A=P Q$ where $P$ is $n \times r, Q$ is $r \times n$ and $r<n$. Any homomorphism maps a non-full matrix to a non-full matrix; if it also maps every full matrix to a full matrix it is called honest. An honest homomorphism is necessarily injective. We recall the following result from ([4], page 283):

In a semifir $R$, the set of all non-full matrices is a prime matrix ideal. It is therefore the least prime matrix ideal, the corresponding epic $R$-field $U$ is the universal field of fractions of $R$ and the natural injection $R \rightarrow U$ is honest. If $\Phi$ is the set of all full matrices over $R$, then $U$ may be obtained as the universal $R$ ring over which all matrices of $\Phi$ are invertible, briefly, $U=R_{\Phi}$ is the universal Ф-inverting ring ([4], page 285).

When there is an epic $R$-field $U$ in which every full matrix over $R$ is invertible (as here) we shall call $U$ the fully inverting ring for $R$; this is necessarily the universal field of fractions of $R$, and the result quoted above tells us that it exists for any semifir. Our first result describes the extension of homomorphisms to fully inverting rings:

Proposition 2.1. Any honest homomorphism between rings $R, R^{\prime}$ wth fully inverting rings $U, U^{\prime}$ extends uniquely to a homomorphism between $U$ and $U^{\prime}$; in particular, any isomorphism between $R$ and $R^{\prime}$ extends to a unique isomorphism between $U$ and $U^{\prime}$.

Proof. Let $\alpha$ be an honest homomorphism from $R$ to $R^{\prime} ; \alpha$ maps the set of full matrices over $R$ to the set of full matrices over $R^{\prime}$, and hence extends uniquely to a homomorphism between their fully inverting rings.

If $\left(K_{\lambda}\right)$ is a family of fields containing a common subfield $k$, then their free product $R={ }_{k}^{*} K_{\lambda}$ is a semifir [2] and so has a universal field of fractions. This will be called the field product of the $K_{\lambda}$ and written ${ }_{k}^{\circ} K_{\lambda}$ or $K_{k}^{\circ} L$ in the case of only two factors. Clearly ' ${ }^{\circ}$ ' is a bifunctor on the category of fields and homomorphisms. This is an instance where the non-commutative case runs more smoothly than the commutative case. The 'free product' (i.e. coproduct) of two commutative $k$ fields $K, L$ in the category of commutative $k$-algebras is given by their tensor product $K \otimes L$. This need not be an integral domain and so need not have a field of fractions, and even though an epic $(K \otimes L)$-field exists, there may be no universal $(K \otimes L)$-field.

We shall also need the fact that the universal field construction and the free product are commuting operations. 
THEOREM 2.2. Let $R$ and $S$ be k-rings (where $k$ is a field) with universal fields of fractions $\bar{R}, \bar{S}$. Then the free product $R_{k}^{*} S$ has a universal field of fractions isomorphic to the field product $\bar{R}_{k}^{\circ} \bar{S}$.

Proof. Clearly $U=\bar{K}_{R}^{\circ} \bar{S}$ is a field of fractions of $R_{k}^{*} S$; we complete the proof by showing that it is universal. Given any epic $(R * S)$-field $K$, we have induced mappings $R \rightarrow K, S \rightarrow K$, obtained by composing $R * S \rightarrow K$ with the natural inclusions. Hence there are specializations $\bar{R} \rightarrow K, \bar{S} \rightarrow K$ which can be combined to a specialization $\bar{R} \circ S \rightarrow K$, necessarily unique, because its values on $R * S$ are prescribed. This shows that $U$ is the universal field of fractions of $R * S$, as claimed.

We observe that this result applies e.g. when $R, S$ are semifirs (containing a field $k$ ).

\section{Subfields of field products}

Let $k$ be a commutative field and $k\langle X\rangle$ the free $k$-algebra on a set $X$. This algebra is a fir and hence has a universal field of fractions [4]. If $K$ is a field generated over a central subfield $k$ by a set $X$, we shall say that $X$ generates $K$ freely over $k$ or that $X$ is a free generating set of $K$ over $k$ if $K$ is the universal field of fractions of the free algebra $k\langle X\rangle$. In this case $K$ itself may also be called a free field over $k$; thus the free field on a single generator $x$ over $k$ is just the rational function field $k(x)$, but for more than one free generator the free field will be noncommutative. Given any field with a central subfield $k$, by a free subset over $k$ we understand a subset $Y$ of the field such that the subfield generated by $Y$ is freely generated by $Y$ over $k$.

We note that a subfield of a purely transcendental (i.e. free commutative) extension need not be free when the transcendence degree exceeds 1 (in degree 1 we have Lüroth's theorem). In the general case little is known about subfields of fields, but it seems not unreasonable to conjecture that every subfield of a free field (over a central subfield $k$ ) is again free over $k$. The proof would probably require a closer analysis of the matrix form for the elements of $K$. Such an attempt, if successful, may also provice techniques for the analysis of field products.

In this section we shall show that every countable field can be embedded in a 2-generator field; this is the analogue of a theorem of Neumann ([8], Theorem 20.7) for groups. As in that case, one may ask whether there is a countable field, or a countably generated field over $k$, containing a copy of every countable field (of a given characteristic), and as for groups, the answer is 'no'. To see this $\left({ }^{*}\right)$ let us denote for any field $K$, by $\mathscr{S}(K)$ the set of isomorphism types of finitely generated subgroups of $K^{*}$, the group of non-zero elements of $K$. If $K$ is countable, then so is $\mathscr{P}(K)$. Now Smith [11] has shown that there are $\mathfrak{c}=2^{\mathrm{N}_{0}}$ isomorphism types of finitely generated orderable groups, and since every countable ordered

(*) I am indebted to A. Macintyre for this proof. 
group can be embedded in a countable field (of prescribed characteristic), it follows that there are $c$ distinct sets $\mathscr{H}(K)$ as $K$ runs over all countable fields of any given characteristic. Therefore these fields cannot all be embedded in a 2-generator field.

Let $P, Q$ be a subrings of a ring $R$ and suppose that $k$ is a common subring of $P$ and $Q$. Then there exists a $k$-bimodule homomorphism

$$
P \otimes_{k} Q \rightarrow R \text {. }
$$

If the mapping (2) is injective, $P$ and $Q$ are said to be linearly disjoint in $R$ over $k$. We observe that this property is not generally symmetric in $P$ and $Q$. With this notion we obtain the following analogue of a lemma of Neumann ([9], cf. [8]), implicitly used in [3].

LEMMA 3.1. Let $P={ }_{K}^{*} R_{\lambda}$ be the free product of a family of $K$-rings, where $K$ is a field. Given a subring $S_{\lambda}$ of each $R_{\lambda}$ and a subfield $k$ of $K$ where $k \subseteq S_{\lambda}$ (for all $\lambda$ ), suppose that for each $\lambda$, the pair $S_{\lambda}, K$ is linearly disjoint in $P$ over $k$. Then the subring $Q$ of $P$ generated by the $S_{\lambda}$ is their free product over $k$.

Proof. Let $B_{\lambda}^{\prime}=\{1\} \cup B_{\lambda}$ be a right $k$-basis for $S_{\lambda}$ (adapted to the subspace $k$ ), then $B_{\lambda}^{\prime}$ is still right $K$-independent, by hypothesis, and can therefore be enlarged to a right $K$-basis $A_{\lambda}^{i}=\{1\} \cup A_{\lambda}$ of $R_{\lambda}$ (still adapted to the subspace $K$ ). Now the non-interacting words in $\bigcup A_{\lambda}$ form a right $K$-basis for $P$; hence the non-interacting words in $\bigcup B_{\lambda}$ are right $k$-independent and they span $Q$. Therefore $Q$ is indeed the free product of the $S_{\lambda}$ over $k$.

We note that the sufficient condition given in this lemma is asymmetric, hence we obtain another sufficient condition by requiring all the pairs $K, S_{\lambda}$ (in that order) to be linearly disjoint over $k$.

Two further results are needed, one on field products, and one on free sets.

LEMMA 3.2. Let $K$ be a field with a subfield $k$, and let $P$ be the field product of $K$ and $k(x)$, where $x$ is an indeterminate centralizing $k$. Then the subfield $Q$ of $P$ generated by the fields $K_{i}=x^{-i} K x^{i}(i \in Z)$ is their field product over $k$.

Proof. Take a family of copies of $K$ indexed by $Z$, say $\left(K_{t}\right)$, and denote by $U$ their field product over $k$. By the universal property of $U$ it follows that $Q$ is a specialization of $U$. From the universal property of $P=K \circ k(x)$, this specialization will be an isomorphism whenever there is some $K$-ring $L$ containing an element $y \neq 0$ such that the specialization of $U$ in $L$ which maps $K_{i} \rightarrow y^{i} K y^{-i}$ is an embedding. Such an $L$ is easily constructed:

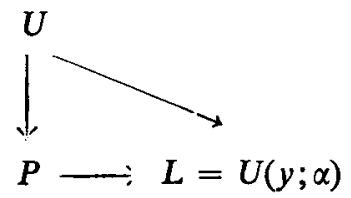


The mapping which takes $K_{i}$ to $K_{i+1}$ is an automorphism of $* K_{i}$ which extends to an automorphism $\alpha$, say, of $U=o K_{i}$. We form the skew polynomial ring $U[y ; \alpha]$ with commutation rule $a y=y a^{\alpha}$. This is an Ore ring and so has a field of fractions $L=U(y, \alpha)$ which has the desired properties.

LEMMA 3.3. Let $E$ be the field generated by a family $\left(e_{i}\right)$ of elements over a central subfield $k$, and $U$ a field freely generated by a family $\left(u_{i}\right)$ over $k$, then the elements $u_{i}+e_{i}$ form a free set in the field product of $U$ and $E$ over $k$.

Proof. The field product $G=E_{k}^{\circ} U$ has the following universal property: given any $E$-field $F$ and any family $\left(f_{i}\right)$ of elements of $F$, there is a unique specialization from $G$ to $F$ (over $E$, with domain generated by $E$ and the $f_{i}$ ) which maps $u_{i}$ to $f_{i}$. In particular, there are specializations from $G$ to itself which map $u_{i}$ to $u_{i}+e_{i}$ (resp. to $u_{i}-e_{i}$ ). On composing these mappings (in either order) we obtain the identity mapping, hence they are inverse to each other, and so are automorphisms. It follows that the $u_{i}+e_{i}$, like the $u_{i}$ form a free set.

We can now prove our main result.

THEOREM 3.4. Let $E$ be a field, countably generated over a central subfield $k$. Then $E$ can be embedded in a field on two generators over $k$.

In essence the proof runs as follows: Suppose that $E$ is generated by $\left(e_{i}\right)$ $(i=0,1, \cdots)$, where $e_{0}=0$. We construct an extension field $L$ generated over $E$ by elements $x, y, z$ satisfying

$$
y^{-i} x y^{i}=z^{-i} x z^{i}+e_{i}
$$

Hence $L$ is in fact generated by $x, y, z$ alone. If we now adjoin $t$ such that $y=t x t^{-1}$, $z=t^{-1} x t$, the resulting field is generated by $x$ and $t$.

To prove Theorem 3.4, let $F_{1}$ be the free field on $x, y$ over $k$; it has a subfield $U$, generated by $u_{i}=y^{-i} x y^{i}(i=0,1, \cdots)$, freely by Lemma 3.2 , and similarly, let $F_{2}$ be the free field on $x, z$ over $k$, with subfield $V$ freely generated by $v_{i}=z^{-i} x z^{i}$ $(i=0,1, \cdots)$.

Let $K$ be the field product of $E$ and $F_{1}$ over $k: K=E \circ F_{1}$. It has a subfield $W$ generated by $w_{i}=u_{i}+e_{i}(i \geqq 0)$, freely by Lemma 3.3. We note that $w_{0}=u_{0}+e_{0}=x_{0}=x$, so $K$ is generated by $x, y$ and the $w_{i}(i \geqq 1)$ over $k$.

Let $L$ be the field product of $K$ and $F_{2}$, amalgamating $W$ and $V$ along the isomorphism $w_{\imath} \leftrightarrow v_{i}$. We note that $w_{0}=x=v_{0}$ and that $L$ is generated by $x, y, z$ and the $w_{i}$, or $x, y, z$ and the $v_{t}$, or simply by $x, y, z$. Now $L$ contains the isomorphic subfields $k\langle x, y\rangle, k\langle z, x\rangle$, hence we can adjoin $t$ to $L$ such that $t^{-1} x t=z, t^{-1} y t$ $=x$ (see [3]), Theorem 6.3). It follows that $L(t)$ so defined is generated by $x$ and $t$ over $k$. This completes the proof of Theorem 3.4.

As in [8] we have the

COROLLARY. Every field can be embedded in a field $L$ such that every countably generated subfield of $L$ is contained in a 2-generator subfield of $L$. 
Proor. Let $E$ be the given field and $E_{\lambda}$ a typical countably generated subfield (over a central subfield $k$, which could of course be the prime field), then there is a 2-generator field $L_{\lambda}$ containing $E_{\lambda}$. Form the field product $M_{\lambda}$ of $E$ and $L_{\lambda}$ over $E_{\lambda} ;$ if we do this for each countably generated subfield of $E$, we get a family $\left(M_{\lambda}\right)$ of fields, all containing $E$. Form their field product $E^{\prime}$ amalgamating $E$; then in $E^{\prime}$ every countably generated subfield of $E$ is contained in a 2-generator subfield of $E^{\prime}$, namely $E_{\lambda}$ is contained in $L_{\lambda}$. Now repeat the process that led from $E$ to $E^{\prime}$ :

$$
E \subseteq E^{\prime} \subseteq E^{\prime \prime} \subseteq \cdots \subseteq E^{\omega} \subseteq E^{\omega+1} \subseteq \cdots \subseteq E^{v},
$$

where $E^{\alpha}=\bigcup\left\{E_{\beta} \mid \beta<\alpha\right\}$ at a limit ordinal $\alpha$, and where $v$ is the first uncountable ordinal. Then $E^{v}$ is a field in which every countably generated subfield is contained .n some $E^{\alpha}(\alpha<v)$ and hence in some 2-generator subfield of $E^{\alpha+1} \subseteq E^{\nu}$. This establishes the corollary.

It is not hard to determine the algebraic elements in a free product. Let $P$ $={ }^{*} R_{\lambda}$ be a free product of a family of integral domains $R_{\lambda}$ over a field $k$, then each $R_{\lambda}$ is a faithfully llat inert extension of $k$, and hence, by Theorem 2.2 of [2], III, $P$ is an integral domain. Suppose that $a \in P$ is right algebraic over $k$, i.e. it satisfies an equation

$$
a^{n} \gamma_{0}+a^{n-1} \gamma_{1}+\cdots+\gamma_{n}=0 \quad\left(\gamma_{l} \in k, \text { not all } 0\right) .
$$

Since $P$ is an integral domain, we may assume that $\gamma_{n} \neq 0$. It follows that

$$
a\left(a^{n-1} \gamma_{0}+\cdots+\gamma_{n-1}\right)\left(-\gamma_{n}^{-1}\right)=1,
$$

and hence $a$ is a unit in $P$. Now any unit in $P$ is a product of units in the factors $R_{\lambda}$ (Theorem 2.2 of [2], III). If $a$ is not in one of the $R_{\lambda}$, then by taking a suitable conjugate, we ensure that $h\left(a^{r}\right)=r h(a)$, where $h$ is the height defined in [2], III. This clearly contradicts (3), hence $a$ lies in some $R_{\lambda}$ and we have proved

THEOREM 3.5. Let $P={ }^{*} R_{\lambda}$ be a free product of (not necessarily commutative) integral domains $R_{\lambda}$ over a field $k$. Then any element right (or left) algebraic over $k$ is conjugate to an element in one of the factors.

Of course it would be more interesting (and presumably also more difficult) to establish the analogue for field products. The conclusion of Theorem 3.5 fails when ditferent subfields are amalgamated. This is shown by the example corresponding to Neumann's [8]: Let $k$ be any commutative field and form the fields $K_{1}=k(x, y), K_{2}=k(y, z), K_{3}=k(z, x)$ with defining relations $y^{-1} x y=x^{-1}$, $z^{-1} y z=y^{-1}, x^{-1} z x=z^{-1}$; it is clear how to construct such fields as fields of fractions of suitable skew polynomial rings. Their free product $P$ exists, with amalgamations $K_{12}=k(y), K_{23}=k(z), K_{31}=k(x)$; this follows as for groups. However, ir $P, x y z$ is an element of order two: $x y z=y x^{-1} z=y z^{-1} x^{-1}$ $=z^{-1} y^{-1} x^{-1}$. Thus $P$ is not even an integral domain. 


\section{Algebraic extensions of skew fields}

In order to be able to build a satisfactory theory of algebraic field extensicns it is necessary to solve the following basic

Problem. Given any field $K$ with a central subfield $k$ algebraically closed in $K$ find an extension field $E$ of $K$ such that any non-constant element $p$ of $K_{k}^{*} k[x]$ vanishes for some value of $x$ in $E$.

Of course it is only necessary to find an extension in which a single equation has a root; then the familiar process will provide an extension in which all equations have solutions. However, there is no guarantee that such an extensicn is unique, nor that all its elements are algebraic over $K$. Now recently, Robinson [10] has outlined a construction which leads to the notion of an algebraically closed skew field; various people have observed that the class of algebraically closed fields constructed in this way constitutes precisely the class of elementary subfields of the homogeneous universal fields constructed by the methods of Jónsson $[6,7]$. Both Jónsson's and Robinson's constructions depend on the fact, proved in [3], that skew fields possess the amalgamation property.

At present we are unable to answer the above problem except in the following special case.

THEOREM 4.1. Let $K$ be a field and $p \in K_{k}^{*} k[x]$ a non-constant polynomial, whose coefficients all lie in a commutative subfield $F$ of $K$. Then there is an extension field $E$ of $K$ in which $p=0$ has a root.

Proof. By hypothesis, $p \in F[x]$ and there is a commutative field $G$ containing $F$ in which $p$ has a zero (e.g. the algebraic closure of $F$ ). Now take $E$ to be the field product of $K$ and $G$ over $F$, then $p$ has a zero in $E$.

By induction we can extend $K$ to a field in which every polynomial with coefficient in some commutative subfield of $K$ has a zero; in particular, in such a field every element has $n$th roots, for every integer $n$.

\section{References}

[1] G. M. Bergman, 'The diamond lemma in ring theory', to appear.

[2] P. M. Cohn, 'On the free product of associative rings', I, Math. Zeits. 71 (1959), 380-398, II. 73 (1960), 433-456, III. J. Algebra 8 (1968), 376-383 (correction 10 (1968), 123.)

[3] P. M. Cohn, 'The embedding of firs in skew fields', Proc. London Math. Soc. (3) 23 (1971), 193-213.

[4] P. M. Cohn, Free rings and their relations (London, New York 1971).

[5] P. M. Cohn, 'Universal skew fields of fractions', Symposia Math. VIII (1972), 135-148.

[6] B. Jónsson, 'Universal relational systems, Math. Scand. 4 (1956), 193-208.

[7] B. Jónsson, 'Homogeneous relational universal systems', Math. Scand. 8 (1960), 137-142. 
[8] B. H. Neumann, 'An essay on free products of groups with amalgamations', Phil. Trans. Roy. Soc. Ser. A 246 (1954), 503-554.

[9] H. Neumann, 'Generalized free products with amalgamated subgroups', I. Amer. J. Math. 70 (1948), 590-625, II. 71 (1949), 491-540.

[10] A. Robinson, 'On the notion of algebraic closedness for noncommutative groups and fields'. J. Symb. Logic 36 (1971), 441-444.

[11] D. B. Smith, 'On the number of finitely generated 0-groups', Pacif. J. Math. 35 (1970), 499-502.

\section{Bedford College}

Regents Park

London NW1 4NS

England. 